\title{
Vitamin D in Cancer and Other Diseases: A New Wonder
}

\section{Biomarker?}

\author{
Dahiya $\mathrm{K}^{1 *}$ and Dhankhar $\mathbf{R}^{2}$ \\ ${ }^{1}$ Department of Biochemistry, Pt. BD Sharma PGIMS, Haryana, India \\ ${ }^{2}$ Department of Radiotherapy, Pt. BD Sharma PGIMS, Haryana, India
}

*Corresponding author: Dr. Kiran Dahiya, 778/28, Bharat Colony, Rohtak, Haryana, India, 124001, Tel: 09896111985, Email: kirandahiya_2002@yahoo.com

\section{Mini Review \\ Volume 2 Issue 4}

Received Date: July 18, 2018

Published Date: August 02, 2018

\begin{abstract}
Vitamin D, a fat soluble hormone, plays a very vital role along with parathyroid hormone and calcitonin in calcium homeostasis. Besides this function, a number of other biologically significant roles attributed to vitamin D include maintenance of health of central nervous system, boosting the immunity and prevention against cancer etc. Besides exerting autocrine activity, this vitamin demonstrates endocrine function also due to its mechanism of action being similar to steroid hormones, capability to get synthesized in the body and its ability to produce effects on distant organs. It is synthesized from 7-dehydrocholesterol in the skin under influence of ultraviolet radiation and gets fully activated by subsequent hydroxylations in liver and kidney to 1, 25-dihydroxycholecalciferol. Recent reports indicate that prevalence of vitamin D deficiency is rampant in general population worldwide necessitating its estimation and supplementation at a large scale. The deficiency of vitamin D might be attributed to the many factors like modernization of lifestyle, improper dietary habits, skin complexion and genetic cause etc. Inadequate levels of vitamin D might play a role in the etiopathogenesis of a number of chronic diseases, important ones being cancer, diabetes, cardiovascular disease and autoimmune disorders etc. which might benefit by including vitamin D supplementation in the treatment. Therefore, on the basis of these properties, it is imperative to assign the status of a biomarker to vitamin D.
\end{abstract}

Keywords: Vitamin D deficiency; Bone health; Calcium homeostasis; Cancer; Diabetes mellitus; Cardiovascular diseases; Autoimmune disorders; Biomarker

Abbreviations: PTH: Parathyroid Hormone; VDR: Vitamin D Receptor; GcMAF: Gc Protein-Derived Macrophage Activating Factor; uPAR: Urokinase Plasminogen Activator; Rb: Retinoblastoma; PCa: Prostate Cancer; BCa: Breast Cancer; PA: Plasminogen Activator;
MMPs: Matrix Metalloproteinases; VEGF: Vascular Endothelial Growth Factor; PGs: Prostaglandins; NFkB: Nuclear Factor Kappa B; CVD: Cardiovascular Disease; RA: Rheumatoid Arthritis; SLE: Systemic Lupus Erythematosus; IBD: Inflammatory Bowel Disease; MS: Multiple Sclerosis. 


\section{Open Access Journal of Cancer \& Oncology}

\section{Introduction}

There is a recent surge in the interest of estimating, analyzing and associating vitamin $\mathrm{D}$ deficiency with a variety of clinical disorders. Vitamin D, a fat-soluble vitamin, is considered a prohormone because the body can synthesize vitamin D from its precursor (7dehydrocholesterol) when exposed to ultraviolet light at a wavelength between 290-315 nm. Its mechanism of action also resembles that of steroid hormones. Active form of vitamin $\mathrm{D}$ is $1 \alpha, 25$-dihydroxycholecalciferol/ $1 \alpha$, 25-dihydroxy vitamin $\mathrm{D}_{3} /$ calcitriol or $1 \alpha, 25(\mathrm{OH})_{2} \mathrm{D}_{3}$. Adequate circulating levels of active form of this vitamin need to be maintained for normal functioning of all the systems of the body irrespective of age and gender. Insufficient levels of vitamin D may affect any system and produce the related clinical abnormality [1]. The potential of vitamin $\mathrm{D}$ for acting as a biomarker depends on its association with an ever increasing number of diseases which are benefitted with vitamin D supplementation [2].

Recently, the scientific evidence is accumulating to demonstrate the insufficiency of vitamin D globally. Factors, which are responsible for the worldwide prevalence of vitamin D insufficiency, may vary among countries; but the principal reason is limitation in either cutaneous synthesis or dietary intake of vitamin D or both. Even the countries where geographical location ensures plenty of sunlight throughout the year like India, vitamin $\mathrm{D}$ deficiency is found to be rampant. This might be a result of poor sun exposure, dark skin complexion, atmospheric pollution, vegetarian foods habits, absence of food fortification with vitamin D and poor intake of vitamin D supplements [3,4].

The main source of vitamin $D_{3}$ is synthesis in presence of sunlight and is virtually considered negligible in the food supply. It is not found in vegetables, fruits or grains and is present in low quantity in meats and other animal food sources, except for fish liver oils and plants such as waxy-leaf nightshade (Solanum glaucophyllum) [2]. Therefore, there is a great market for supplements of vitamin $\mathrm{D}$ available in different forms and with different names which are being increasingly prescribed in addition to the standard treatment regimens [5].

To understand the role of vitamin D deficiency in various health disorders, it is imperative to assess its role in human physiology and biochemistry. The physiological functions of active vitamin $\mathrm{D} \quad(1 \alpha, 25-$ dihydroxycholecalciferol) mainly pertain to calcium homeostasis along with maintenance of health of diverse organs [6].

\section{Vitamin $\mathrm{D}_{2}$ versus Vitamin $\mathrm{D}_{3}$}

The natural form of vitamin D in all animals and the form synthesized in human skin on exposure to sunlight is cholecalciferol, vitamin $\mathrm{D}_{3}$. Ergocalciferol (vitamin $\mathrm{D}_{2}$ ) is a synthetic product derived by irradiation of plant sterols/ergosterol. Initially, the two forms of the vitamin were considered to be interchangeable and equivalent, however, since the availability of the measurement of serum $25(\mathrm{OH}) \mathrm{D}_{3}$ as an indicator of vitamin $\mathrm{D}$ functional status, it has become clear that vitamin $\mathrm{D}_{2}$ is substantially less potent than vitamin $\mathrm{D}_{3}$. Both of these are absorbed from the intestine and get hydroxylated at $25^{\text {th }}$ position in liver. However, vitamin $\mathrm{D}_{2}$ leads to upregulation of 24hydroxylase, converting the administered $\mathrm{D}_{2}$ and endogenous $\mathrm{D}_{3}$ to their inactive forms. Thus, administration of vitamin $\mathrm{D}_{2}$ seems to have no advantage over vitamin $\mathrm{D}_{3}$, which is the natural form of the vitamin and is less expensive [7].

\section{Assessment of vitamin D}

Ideally, assessment of vitamin $\mathrm{D}$ status is recommended only for those at risk for vitamin D deficiency. But due to widespread prevalence of deficiency in general population, assessment of vitamin D status is increasingly being advised to people who present with unexplained clinical features and are found not responding to treatment. The levels of 25hydroxycholecalciferol are commonly analyzed though $1 \alpha, 25$-dihydroxycholecalciferol is the active form of vitamin D. This is because the serum $25(\mathrm{OH}) \mathrm{D}_{3}$ is the major circulating form of vitamin $\mathrm{D}$ and has a longer halflife (2 to 3 weeks) as compared to $1 \alpha, 25(\mathrm{OH})_{2} \mathrm{D}_{3}$ (3- 4 hours). The concentration of $1 \alpha, 25(\mathrm{OH})_{2} \mathrm{D}_{3}$ is 1,000 times lower than that of $25(\mathrm{OH}) \mathrm{D}_{3}$ in circulation. Another fact needing consideration is that vitamin $\mathrm{D}$ deficiency is associated with a compensatory increase of parathyroid hormone secretion, which in turn, stimulates the kidneys to produce more $1 \alpha, 25$-dihydroxycholecalciferol. Thus, when $25(\mathrm{OH}) \mathrm{D}_{3}$ levels fall due to vitamin D deficiency, the concentrations of $1 \alpha, 25(\mathrm{OH})_{2} \mathrm{D}_{3}$ may still remain within normal limits or sometimes even might be elevated. Though in patients of suspected renal impairment and inherited metabolic disorders of vitamin $\mathrm{D}$ and phosphate metabolism, both the forms should be measured [8].

\section{Reference Range for Vitamin D}

Normal vitamin D levels range between 30-100 $\mathrm{ng} / \mathrm{mL}$. Vitamin D deficiency is generally recognized as a $25(\mathrm{OH}) \mathrm{D}_{3}$ level $<20 \mathrm{ng} / \mathrm{mL}$ while vitamin D insufficiency 


\section{Open Access Journal of Cancer \& Oncology}

has been defined as a serum $25(\mathrm{OH}) \mathrm{D}_{3}$ level of 21 to 29 $\mathrm{ng} / \mathrm{mL}$. As Vitamin D is a fat soluble vitamin, toxicity may appear at levels $>150 \mathrm{ng} / \mathrm{mL}$ while levels $>100 \mathrm{ng} / \mathrm{ml}$ are considered excess levels [7].

\section{Toxicity}

Vitamin D, being fat soluble, gets stored in the body and is capable of producing serious toxic effects, including death. An excess of vitamin D causes hypercalcemia, which can cause over calcification of the bones, soft tissues, heart and kidneys as well as hypertension. Symptoms of vitamin D toxicity may include dehydration, vomiting, decreased appetite, irritability, constipation, fatigue, muscle weakness and metastatic calcification of the soft tissues and tend to appear several months after excessive intake of vitamin D. Treatment includes a lowcalcium diet along with corticosteroid drugs and a full recovery may be expected within a month [7].

\section{Functions of vitamin D}

\section{Bones and Teeth}

Vitamin D plays a central role in homeostasis of calcium, a mineral needed by bones, teeth and other tissues. It increases serum calcium concentrations through 3 separate actions. First and foremost, it induces the proteins involved in active intestinal calcium absorption and also stimulates active intestinal absorption of phosphate. Second, it maintains blood calcium concentrations in the normal range even when no calcium is taken in diet. Vitamin D stimulates osteoblasts to produce receptor activator nuclear factor-kappa B ligand (RANKL). RANKL, then, stimulates osteoclastogenesis and activates resting osteoclasts for bone resorption. It is very important to note, however, that, in vivo, both vitamin $\mathrm{D}$ and parathyroid hormone (PTH) are required for action. Third, it increases reabsorption of filtered calcium in the distal renal tubule. Again, both PTH and the vitamin D hormone are required. While vitamin $\mathrm{D}$ is responsible for reabsorption of both calcium and phosphate, PTH leads to reabsorption of only calcium promoting phosphate excretion [9]. Calcium is essential to maintain strong bones and teeth, not only in growing children and adolescents, but also in adults. Low vitamin D levels can cause bones to become soft and brittle due to low calcium content, increasing the risk of fractures. This condition is called rickets in growing children and, in adults, osteomalacia. Low vitamin $\mathrm{D}$ can also contribute to osteoporosis, in which the bones become thin and brittle [10]. Intestinal absorption of dietary calcium and phosphorus is decreased with vitamin D deficiency, which causes an increase in parathyroid hormone levels [9].

\section{Other Functions}

Research has shown that vitamin D receptor (VDR) is found throughout the central nervous system including the hippocampus region of the brain responsible for both memory and emotion. Vitamin D is responsible for the regulation of enzymes located in the brain and cerebrospinal fluid which aid in nerve growth, neurotransmitter production and synaptic density of neurons. It also protects nervous tissue from oxidative stress, controls the immune system response and is involved in the homeostatic processes of intracellular calcium and phosphorus levels. Vitamin D deficiency, especially during neonatal development, is believed to increase the risk of schizophrenia, multiple sclerosis and other diseases of the central nervous system [11].

Another important organ for significant role of vitamin $D$ is the immune system. Vitamin D deficiency affects the immune system, especially $\mathrm{T}$ cell-mediated immunity, whereas vitamin $D$, in excess, actually suppresses certain aspects of the immune system [12]. Besides this, serum vitamin D levels have been correlated to muscle cell contractility and muscle strength. Low serum levels have been related to proximal muscle weakness, gait disturbance, paresthesia and discomfort within the muscles. The expression of VDR and its interaction at the molecular level with proteins that are involved in inflammation, signaling and ultimately contractile function of both skeletal and cardiac muscle is of utmost significance [13].

\section{Vitamin D and Chronic Diseases}

Rickets and osteomalacia are the classical vitamin D deficiency diseases but as the vitamin $\mathrm{D}$ has been shown to perform a wide spectrum of functions, its deficiency is bound to be associated with other diseases also.

Here is a brief account of different chronic diseases associated with inadequate vitamin D levels in the body:

\section{Osteoporosis}

The role of vitamin $\mathrm{D}$ in the pathogenesis and course of osteoporosis involves its role in calcium homeostasis and the autocrine activity of the vitamin. It is not surprising that most of the clinical trials showing fracture prevention with calcium supplementation have involved treatment with vitamin $\mathrm{D}$ as well. In addition, apparently through an autocrine pathway, vitamin $\mathrm{D}$ has been shown to reduce fall risk within only a few weeks of initiating the treatment, in some trials by as much as $50 \%$. It is likely 


\section{Open Access Journal of Cancer \& Oncology}

that this effect is partly responsible for the reduced fracture risk observed in treatment studies $[8,12,14]$.

\section{Cancer}

There is substantial epidemiologic data available to prove an inverse association between incident cancer risk and antecedently measured serum vitamin D. Vitamin D deficiency is reported to present increased risk for various cancers including prostate, colon, breast, lung, thyroid along with leukemia and lymphoma. Reports are there to suggest that besides possessing anti-tumor properties, vitamin $\mathrm{D}$ regulates expression of genes involved in the multiplication and spread of cancer. An important immune supporting protein, Gc proteinderived macrophage activating factor (GcMAF), which requires vitamin $\mathrm{D}$ for its synthesis, is vital to an appropriate immune response leading to eradication of tumors. Therefore, ensuring a sufficient supply of vitamin D for the synthesis of GcMAF is an effective strategy to inhibit tumor growth. GcMAF acts by inhibiting the receptor for urokinase plasminogen activator (UPAR), responsible for tumor [15]. Vitamin D is also hypothesized to reduce the risk of breast cancer by inhibiting cell proliferation via the nuclear vitamin D receptor (VDR). Nucleotide polymorphisms (SNP) in the $V D R$ gene have been inconsistently associated with breast cancer risks [16].

\section{Various Mechanisms for Role of Vitamin D in Cancer}

A number of mechanisms have been put forward to explain the anti-cancer actions of vitamin D [17].

\section{Inhibition of Cell Proliferation}

In most cell types that express a functional VDR, exposure to $1,25(\mathrm{OH})_{2} \mathrm{D}_{3}$ results in the accumulation of cells in the G0/ G1 phase of the cell cycle. The transition from $\mathrm{G}$ to $\mathrm{S}$-phase is controlled by the pocket proteins retinoblastoma (Rb), p107 and p130. The phosphorylation status of these proteins determines their association with members of the E2F family of transcriptional regulators that play a pivotal role in mediating gene expression during cell proliferation $[16,17]$.

\section{Induction of Apoptosis}

Vitamin D can trigger apoptosis though the effect is not uniform for all types of cells. Depending on the cell type, $1 \alpha, 25(\mathrm{OH})_{2} \mathrm{D}_{3}$ may decrease the expression of the anti-apoptotic factors (Bcl-2, Bcl- XL) or/and may increase the pro-apoptotic equivalents (Bax, Bak), inducing apoptosis . In prostate cancer (PCa) and breast cancer (BCa) cells, vitamin $\mathrm{D}$ activates the intrinsic pathway of apoptosis causing the disruption of mitochondrial function, cytochrome release and production of reactive oxygen species. In some cells $1 \alpha, 25(\mathrm{OH})_{2} \mathrm{D}_{3}$ directly activates caspases to induce apoptosis $[17,18]$.

\section{Inhibition of Spread of Tumor}

The mechanisms underlying this effect include the inhibition of angiogenesis and the regulation of the expression of key molecules involved in invasion and metastasis like the components of the plasminogen activator (PA) system and matrix metalloproteinases (MMPs), decreasing the expression of tenascin-C, an extracellular matrix protein that promotes growth, invasion and angiogenesis, down-regulation of the expression of $\alpha 6$ and $\beta 4$ integrins and increase in the expression of E-cadherin, a tumor suppressor gene whose expression is inversely correlated to metastatic potential as well as reducing the vascular endothelial growth factor (VEGF)-induced endothelial cell growth and elongation [19].

\section{Anti-inflammatory Effect}

Vitamin D leads to the inhibition of the synthesis of prostaglandins (PGs) and their biologic actions by decreasing the expression of gene for the enzyme cyclooxygenase- 2 and increasing the synthesis of its catabolic enzyme 15-hyroxyprostaglandin dehydrogenase. Vitamin D also suppresses the activation and signaling of nuclear factor kappa $B$ (NFkB), a transcription factor required to regulate the expression of genes involved in inflammatory and immune responses and cellular proliferation leading to carcinogenesis $[20,21]$.

\section{Miscelleneous Roles}

Other mechanism suggested for role of vitamin D against cancer is that vitamin $\mathrm{D}$ and its metabolites produce inhibition of tumor angiogenesis, stimulation of mutual adherence of cells and promotion of intercellular communication through gap junctions strengthening the antagonism of property of contact inhibition. Exposure to typical carcinogens, furthermore, predisposes to development of cancer in experimental systems. This has been demonstrated both for animals with knockout of the vitamin $\mathrm{D}$ receptor and for animals with induced, nutritional vitamin $\mathrm{D}$ deficiency [22]. There are reports to suggest that expression of VDR and $1 \alpha, 25(\mathrm{OH})_{2} \mathrm{D}_{3}$ - 


\section{Open Access Journal of Cancer \& Oncology}

associated gene signature in stromal fibroblasts predicts a favourable clinical outcome in colorectal carcinoma [23].

\section{Role in Treatment and Outcome of Cancer}

Vitamin D status may have a significant bearing on cancer outcome also as higher mortality in patients with colorectal carcinoma was found to be associated with groups having lower vitamin D levels. Chemotherapy for colorectal rectal carcinoma has also been reported to induce a state of vitamin D insufficiency or deficiency [24]. Similarly, increased radiosensitization has been reported in prostate carcinoma cells with vitamin D/ vitamin $\mathrm{D}$ analogue supplementation. $1 \alpha, 25(\mathrm{OH})_{2} \mathrm{D}_{3}$ has been reported to be effective against adverse effects associated with radiotherapy as vitamin D deficiency has been found associated with increased severity of radiation-induced acute proctitis in cancer patients [25]. Thus, vitamin D plays a quite significant role at every stage of cancer as well as during treatment.

\section{Immunity/Response to Infection}

Response to infection is not found to be appropriate with suboptimal vitamin D status. Earlier when rickets was quite prevalent, children with this disorder were frequently found to die of respiratory infections. Vitamin $\mathrm{D}$, in its autocrine role, has been reported to play an important role in various aspects of the immune response. Macrophages exert better phagocytic activity in individuals who received vitamin D supplementation [26].

$1 \alpha, 25$-dihydroxycholecalciferol or calcitriol has been identified as a potent modulator of macrophage functions as well as that of B- and T-lymphocyte mediated immune responses. Macrophages not only express VDR, but are also endowed with CYP27B1 activity. But under conditions of insufficient serum 25-hydroxy vitamin $\mathrm{D}_{3}$ levels, only limited amount of calcitriol can be produced by macrophages. The resulting impairment of macrophage activation and function might explain the high prevalence of infectious diseases in children with rickets [27].

\section{Diabetes}

Vitamin D deficiency is found associated with both type 1 and type 2 diabetes mellitus. Vitamin D possesses immunomodulatory properties also. The autoimmune nature of type $1 \mathrm{DM}$ has been declared with the detection of auto-antibodies against islet $\beta$-cells and their infiltration by $\mathrm{T}$ cells, $\mathrm{B}$ cells and macrophages. A relationship between sunlight exposure and the incidence of type 1 DM has been reported by some authors $[1,5]$. Substantial evidence is there to suggest that vitamin D deficiency in early life accelerates the appearance of autoimmune diabetes mellitus and that vitamin D supplementation can prevent the development of the disease. It has been known for a long time that $1 \alpha, 25$ dihydroxycholecalciferol is a positive regulator of insulin secretion by pancreatic $\beta$ cells. These cells are endowed with CYP27B1 activity and thus, low 25-hydroxy vitamin $D$ levels are associated with impaired $\beta$-cell function [28].

Vitamin D receptors in pancreatic $\beta$-cells play an important role in the progression of type $2 \mathrm{DM}$. Vitamin D deficiency is related to insufficient insulin secretion, insulin resistance and $\beta$-cell dysfunction in the pancreas [29].

\section{Hypertension and Cardiovascular Disease (CVD)}

There is a strong association between vitamin D deficiency and hypertension. Vitamin D causes direct suppression of the renin-angiotensin system by acting as a negative endocrine regulator of renin gene expression in vivo. It decreases intimal thickening of blood vessels by inhibiting the accumulation of extracellular matrix within the inner vessel wall through its inhibitory effect on matrix metalloproteinases. In addition, vitamin D decreases arterial stiffness by causing upregulation of nitric oxide synthesis in endothelium. Thus, vitamin D helps in preventing hypertension as well as cardiovascular diseases [30]. Interestingly, vitamin D receptor has been identified on the surface of smooth muscle cells, endothelial cells, myocardial cells, inflammatory cells and in platelets, thus, potentially influencing thrombosis. An independent relationship has been observed between vitamin D deficiency and cardiovascular risk factors like hypertension, diabetes mellitus, obesity, metabolic syndrome, subclinical atherosclerosis and coronary calcification. An association of vitamin $\mathrm{D}$ deficiency has been found with cardiovascular events such as myocardial infarction, congestive heart failure and sudden cardiac death [31].

\section{Autoimmune Disorders}

Vitamin D deficiency has been found to be associated with autoimmune diseases including rheumatoid arthritis (RA), systemic lupus erythematosus (SLE), inflammatory bowel disease (IBD) and multiple sclerosis (MS) while vitamin $\mathrm{D}$ supplementation prevents the onset and/or development of these diseases [4]. Insufficient vitamin D levels have been observed in patients with Hashimoto's 


\section{Open Access Journal of Cancer \& Oncology}

thyroiditis, an autoimmune thyroid disease. It is interesting to note that both vitamin $\mathrm{D}$ and thyroid hormone bind to similar receptors called steroid hormone receptors. A mutant gene in the vitamin D receptor was shown to predispose people to autoimmune thyroid disease including Graves' disease and Hashimoto's thyroiditis [32].

\section{Future Perspectives}

It can conveniently be claimed that this article is able to achieve only the tip of the iceberg. There is a possibility for the diseases discussed above that vitamin D deficiency rather than being directly causal, may act by interfering the ability of the tissues concerned to deal adequately with both physiologic stimuli and pathologic signals. The actual etiopathogenesis of these chronic diseases, which are multifactorial in nature, cannot be elucidated until the widespread problem of vitamin D deficiency has been corrected. For many of the diseases, clinical features might be representative of insufficient vitamin D status as well as the actual disorder. To be able to deal with this question should be the aim of the future research in this direction.

Therefore, with the potential to play diverse role in so many major clinical disorders, it is just appropriate to term vitamin $\mathrm{D}$ a new biomarker.

\section{References}

1. Holick MF (2007) Vitamin D deficiency. N Engl J Med 357(3): 266-281.

2. Kumar M, Kumar G, Verma VK, Gupta M, Gautam RK (2017) A study of association of vitamin D deficiency and coronary artery disease. Int J Res Med Sci 5(5): 1765-1770.

3. Harinarayan CV, Joshi SR (2009) Vitamin D status in India-its implications and remedial measures. J Assoc Physicians India 57: 40-48.

4. Hagenau T, Vest R, Gissel TN, Poulsen CS, Erlandsen M, et al. (2009) Global vitamin D levels in relation to age, gender, skin pigmentation and latitude: an ecologic meta-regression analysis. Osteoporos Int 20(1): 133-140.

5. Tiwari P, Sharma N (2017) Role of Vitamin D in Various Illnesses: A Review. J Pharma Care Health Sys 4(3): 176.
6. Martineau A, Jolliffe D (2014) Vitamin D and Human Health: from the Gamete to the Grave: Report on a meeting held at Queen Mary University of London, 23rd-25th April 2014. Nutrients 6(7): 2759-2919.

7. Alshahrani F, Aljohani N (2013) Vitamin D: Deficiency, Sufficiency and Toxicity. Nutrients 5(9): 3605-3616.

8. Heaney RP (2008) Vitamin D in Health and Disease. Clin J Am Soc Nephrol 3(5): 1535-1541.

9. Suda T, Ueno Y, Fujii K, Shinki T (2003) Vitamin D and bone. J Cell Biochem 88(2): 259-266.

10. Looker AC, Mussolino ME (2008) Serum 25hydroxyvitamin D and hip fracture risk in older U.S. white adults. J Bone Miner Res 23(1): 143-150.

11. Eyles DW, Liu PY, Josh P, Cui X (2014) Intracellular distribution of the vitamin $\mathrm{D}$ receptor in the brain: comparison with classic target tissues and redistribution with development. Neuroscience 268: 1-9.

12. Zella JB, DeLuca HF (2003) Vitamin D and autoimmune diabetes. J Cell Biochem 88(2): 216-222.

13. Polly P, Tan TC (2014) The role of vitamin D in skeletal and cardiac muscle function. Front Physiol 5: 145.

14. Bischoff-Ferrari HA, Dawson-Hughes B, Willett WC, Staehelin HB, Bazemore MG, et al. (2004) Effect of vitamin D on falls. JAMA 291(16): 1999-2006.

15. Kanda S, Mochizuki Y, Miyata Y, Kanetake H, Yamamoto N (2002) Effects of Vitamin D3-Binding Protein-Derived Macrophage Activating Factor (GcMAF) on Angiogenesis. J Natl Cancer Inst 94(17): 1311-319.

16. Garland CF, Garland FC, Gorham ED, Lipkin M, Newmark H, et al. (2006) The role of vitamin D in cancer prevention. Am J Public Health 96(2): 252261.

17. Dhankhar R, Dahiya K, Ahlawat R, Dahiya P, Singh S, et al. (2018) A review of the role portrayed by vitamin D in cancer. Cancer Ther Oncol Int J 11(2): 555807.

18. Zheng Y, Trivedi T, Lin RCY, Fong-Yee C, Nolte R, et al. (2017) Loss of the vitamin D receptor in human breast and prostate cancers strongly induces cell 


\section{Open Access Journal of Cancer \& Oncology}

apoptosis through downregulation of Wnt/ $\beta$-catenin signaling. Bone Res 5: 17023.

19. Grant WB (2008) Vitamin D may reduce prostate cancer metastasis by several mechanisms including blocking Stat3. Am J Pathol 173(5): 1589-1590.

20. Mantovani A, Allavena P, Sica A, Balkwill F (2008) Cancer-related inflammation. Nature 454(7203): 436444.

21. Maeda S, Omata M (2008) Inflammation and cancer: role of nuclear factor-kappa B activation. Cancer Sci 99(5): 836-842.

22. Pilz S, Kienreich K, Tomaschitz A, Ritz E, Lerchbaum E, et al. (2013) Vitamin D and cancer mortality: systematic review of prospective epidemiological studies. Anticancer Agents Med Chem 13: 107-117.

23. Ferrer-Mayorga G, Gómez-López G, Barbáchano A, Fernández-Barral A, Peña C, et al. (2017) Vitamin D receptor expression and associated gene signature in tumour stromal fibroblasts predict clinical outcome in colorectal cancer. Gut 66(8):1449-1462.

24. Fakih MG, Trump DL, Johnson CS, Tian L, Muindi J, et al. (2009) Chemotherapy is linked to severe vitamin D deficiency in patients with colorectal cancer. Int J Colorectal Dis 24(2): 219-224.

25. Ghorbanzadeh-Moghaddam A, Gholamrezaei A, Hemati S (2015) Vitamin D deficiency is associated with the severity of radiation-induced proctitis in cancer patients. Int J Radiat Oncol Biol Phys 92(3): 613-618.

26. Cannell JJ, Vieth R, Umhau JC, Holick MF, Grant WB, et al. (2006) Epidemic influenza and vitamin D. Epidemiol Infect 134(6): 1129-1140.

27. Adams JS, Hewison M (2012) Extrarenal Expression of the 25-Hydroxyvitamin D-1-Hydroxylase. Arch Biochem Biophys 523(1): 95-102.

28. Kasper LH, Reder AT (2014) Immunomodulatory activity of interferon-beta. Ann Clin Transl Neurol 1(8): 622-631.

29. Pittas A, Lau J, Hu F, Dawson-Hughes B (2009) The role of vitamin $\mathrm{D}$ and calcium in type 2 diabetes. A systematic review and meta-analysis. J Clin Endocrinol Metab 92(6): 2017-2029.

30. Aggarwal R, Akhthar T, Jain SK (2016) Coronary artery disease and its association with Vitamin D deficiency. J Midlife Health 7(2): 56-60.

31. Pilz S, März W, Wellnitz B, Seelhorst U, FahrleitnerPammer A, Dimai HP, et al. (2008) Association of vitamin $\mathrm{D}$ deficiency with heart failure and sudden cardiac death in a large cross-sectional study of patients referred for coronary angiography. J Clin Endocrinol Metab 93(10): 3927-3935.

32. Adorini A, Penna G (2008) Control of autoimmune diseases by the vitamin $\mathrm{D}$ endocrine system. Nat Clin Pract Rheumatol 4(8): 404-412.

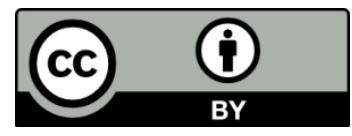

